\title{
Activin A enhances MMP-7 activity via the transcription factor AP-1 in an esophageal squamous cell carcinoma cell line
}

\author{
KEIJI YOSHINAGA* , KOSHI MIMORI*, HIROSHI INOUE, YUKIO KAMOHARA, \\ KEISHI YAMASHITA, FUMIAKI TANAKA and MASAKI MORI \\ Department of Molecular and Surgical Oncology, Medical Institute of Bioregulation, Kyushu University, Beppu, Japan
}

Received January 28, 2008; Accepted March 27, 2008

DOI: 10.3892/ijo_00000027

\begin{abstract}
Activin A, a member of the transforming growth factor $B$ (TGF- $B$ ) superfamily, is often overexpressed in solid carcinomas. We have previously reported that the expression of activin A is associated with lymph node metastasis in esophageal cancer. In the current study, our goal was to clarify the molecular mechanisms underlying the aggressive behavior of tumors expressing high levels of activin A. Using cDNA microarrays, the gene expression profile of a human esophageal carcinoma cell line (KYSE170) stably transfected with activin $\beta A$ (Act- $\beta A$, a subunit of activin A) was compared with those of control human esophageal carcinoma cell lines. We found that expression of MMP-7 was higher in the Act- $\beta$ A transfectants than in the control cells. To reveal the mechanism of expression of MMP-7 mediated by activin A, we evaluated mRNA expression of MMP-7 and Act-BA with or without activin A neutralizing antibody, using real-time PCR and Northern blot analysis. We also performed promoter analysis of the MMP-7 promoter and assessed c-Jun and Smad2/3 expression. MMP-7 expression in the transfectants was correlated with the level of Act- $B A$ expression and was reduced by activin $A$ neutralizing antibody. The Act- $B A$ transfectants had higher MMP-7 promoter activity than control cells. MMP-7 promoter activity was not affected by mutation in the Smad binding site, while mutation of the AP-1 binding site did reduce activity. Moreover, the expression of c-Jun was increased in Act- $B A$ transfectants. These results indicate that activin A may modulate the expression of MMP-7 via AP-1 and not through $\mathrm{Smad} 2 / 3$.
\end{abstract}

Correspondence to: Dr Masaki Mori, Department of Molecular and Surgical Oncology, Medical Institute of Bioregulation, Kyushu University, 4546 Tsurumibaru, Beppu 874-0838, Japan

E-mail: mmori@beppu.kyushu-u.ac.jp

${ }^{*}$ Contributed equally

Abbreviations: TGF- $\mathrm{B}$, transforming growth factor beta; RT-PCR, reverse transcription polymerase chain reaction; MMP-7, matrix metalloproteinase 7; Act- $\mathrm{BA}$, activin beta A

Key words: activin A, MMP-7, AP-1, esophageal carcinoma

\section{Introduction}

We previously reported that overexpression of activin $\mathrm{A}$ in esophageal carcinoma tissues is associated with lymph node metastasis (1). Other studies have shown elevated activin A expression in pancreatic (2), prostate (3), ovarian (4) and colon (5) carcinomas. Moreover, patients with endometrial and cervical carcinomas have high serum levels of activin A (6), and activin A also stimulates the growth of BALB/c 3T3 fibroblasts, granulosa cells and ovarian carcinoma cell lines (7-9). Interestingly, mice deficient in inhibin A, which is a specific inhibitor of activin A, exhibit high levels of circulating activin and develop gonadal stromal tumors, raising the possibility that increased activin A expression may be tumorigenic under certain circumstances (10).

In the current study, we used an esophageal carcinoma cell line (KYSE170) stably transfected with activin BA (Act$B A)$, a subunit of activin $A$, and investigated the gene expression profiles using cDNA microarray analysis. We show that Act- $\beta A$ transfection significantly increased MMP-7 expression levels. MMP-7 is primordial member of the MMP family (11). It can degrade laminin (12), type IV collagen (13) and entactin (14), which are the main components of the basement membrane, and activate other important MMPs (MMP-1, MMP-2 and MMP-9) $(15,16)$. It also inactivates $\alpha 1$-antitrypsin (17), which augments serine protease activity, and thus activates MMPs indirectly. We have demonstrated previously a correlation between MMP-7 and both tumor progression and metastasis in the human colon (18), gastric carcinoma (19) and esophageal carcinoma (20). The promoter of MMP-7 has a TATA box and binding sites for Ets, TCF, Smad and AP-1 (21). To better understand the role of activin A, we evaluated the expression of MMP-7 in the presence or absence of activin A neutralizing antibody. Moreover, we performed MMP-7 promoter assays with or without a mutation in Smad or AP-1 binding sites. Finally, we assessed the location of Smad2/3 and c-Jun proteins by immunoblot and immunofluorescent staining.

\section{Materials and methods}

Cell lines and activin- $\beta A$ (Act- $\beta A$ ) transfection. The esophageal carcinoma cell line KYSE170 was obtained from the Department of Surgery and Surgical Basic Science, Kyoto University (Kyoto, Japan). KYSE170 was stably transfected 


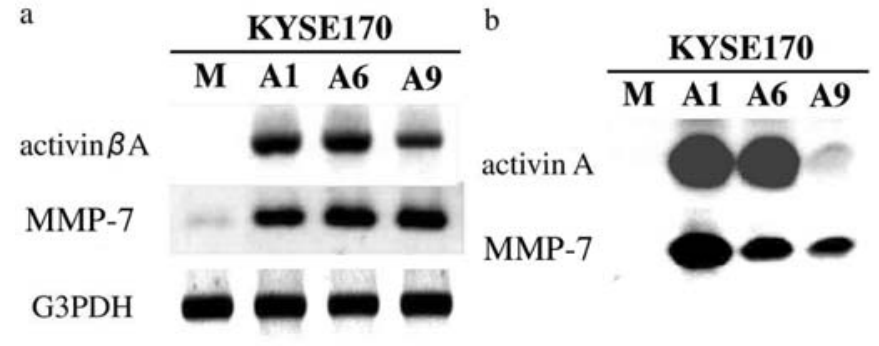

Figure 1. Expression of Act- $B A$ and MMP-7 assessed by (a) Northern blot and (b) immunoblot. (a) Expression level of $28 \mathrm{~S}$ were quite similar in the three transfected clones (KYSE170A1, 6 and 9) and in the control cells (KYSE170M). Expression of Act-BA was not detected in KYSE170M cells, but was observed in KYSE170A1, A6 and A9 cells. A faint band of MMP-7 was detected in KYSE170M cells. The stronger bands were detected in the three transfected clones (KYSE170A1, 6 and 9). (b) The immunoblot failed to detect expression of activin A and MMP-7 in KYSE170M cells. However, expression of activin A and MMP-7 is detected in KYSE170A1, 6 and 9 cells.

with Act- $\beta A$. The PCR product of full-length Act- $\beta A$ cDNA was ligated into the pcDNA 3.1 expression vector (Invitrogen, Inc., Carlsbad, CA). It was confirmed that no mutation had occurred. Following transient transfection with Act-ßA using lipofectamine (Life Technologies, Inc., Rockville, MD), stable Act- $\beta A$ transfectants were selected using geneticin (G418; Life Technologies). The expression of Act- $B A$ mRNA was confirmed by both Northern blot and immunoblot (Fig. 1). All cell lines were maintained in RPMI1640 (Nissui Pharmaceutical, Tokyo, Japan) supplemented with $10 \%$ fetal bovine serum (Equitech-Bio, Inc., Kerrville, $\mathrm{TX})$. We isolated three single clones of Act-BA cDNA transfectant which express different levels of activin A.

\section{RNA extraction and Northern blotting}

Total RNA isolation. Subconfluent cultured cell lines in a state of subconfluency were homogenized in $0.85 \mathrm{M}$ guanidine thiocyanate, and total RNA was obtained by ultracentrifugation through a cesium chloride cushion, as described previously (22).

RNA analysis. Equal amounts $(15 \mu \mathrm{g})$ of total cellular RNA were applied to lanes of a $1.2 \%$ agar-formaldehyde gel and electrophoresed for $7 \mathrm{~h}$. The RNA samples were then transferred to nylon membranes (Hybond-N+; Amersham Pharmacia Biotech UK, Inc., Little Chalfont, UK). The membranes were cross-linked with $120,000 \mathrm{~mJ} / \mathrm{cm}^{2}$ using a UV Stratalinker 1800 (Stratagene, Inc., La Jolla, CA). After overnight hybridization at $42^{\circ} \mathrm{C}$, the blots were washed at a final stringency of $0.1 \mathrm{X} \mathrm{SSPE}$ and $0.1 \% \mathrm{SDS}$ at $65^{\circ} \mathrm{C}$. Autoradiography was performed at room temperature with an identifying screen using a Bio-Image analyzer BAS 2500 (Fuji Photo Film Co., Tokyo, Japan).

Probe preparation. The DNA probes for the open reading frame of Act- $\beta \mathrm{A}$ were subcloned into the pCR II cloning vector (Invitrogen), digested with EcoRI, loaded onto $1.0 \%$ agar gels and electrophoresed. The inserts were extracted using a gel extraction kit (Takara, Inc., Otsu, Japan) and labeled with $\left.{ }^{32} \mathrm{P}\right]$-deoxycytosine triphosphate using a random- primed DNA labeling kit (Takara). A DNA probe for glyceraldehyde-3-phosphate dehydrogenase (G3PDH) was made as previously described (23).

Quantitative reverse transcription polymerase chain reaction. cDNAs were synthesized from $8 \mu \mathrm{g}$ of total RNA in $30 \mu 1$ reaction mixtures, as described previously (24). The products were loaded onto $1.2 \%$ agarose gels with $400 \mathrm{ng} / \mathrm{ml}$ ethidium bromide and electrophoresed. Realtime, no-nested PCR for Act- $\beta A$ and MMP-7 cDNA (bp) was performed using a LightCycler thermal cycler system (Roche Diagnostics, Basel, Switzerland), according to the manufacturer's instructions. The following primers were used: Act- 3 A sense, 5'-TGG GCA AGA AGA AGA AGA AAG-3', and antisense, 5'-CGC AGT AGT TGG CAT GAT AGC-3'. For MMP-7, the primers were: sense, 5'-TAC AGT GGG AAC AGG CTC AGG-3', and antisense, 5'-GGC ACT CCA CAT CTG GGC T-3'. As previously described (25), for G3PDH we used sense, 5'-TTG GTA TCG TGG AAG GAC TCA-3', and antisense, 5'-TGT CAT CAT ATT TGG CAG GTT T-3'. For PCR, $1 \mu 1$ of RNA was placed into a $19 \mu 1$ reaction volume containing $0.67 \mu 1$ primer, $1.2 \mu 125 \mathrm{mM}$ $\mathrm{MgCl}_{2}$, and $2 \mu \mathrm{l} \mathrm{LightCycler-FastStart} \mathrm{DNA} \mathrm{Master}$ SYBR-Green I mix (Roche Diagnostics). The protocol included a polymerase activation step at $95^{\circ} \mathrm{C}$ for $600 \mathrm{sec}$ followed by 40 cycles that each included $95^{\circ} \mathrm{C}$ denaturation for $20 \mathrm{sec}, 55^{\circ} \mathrm{C}$ annealing for $20 \mathrm{sec}$ and $72^{\circ} \mathrm{C}$ extension for $20 \mathrm{sec}$. Negative controls were run concomitantly to confirm that the samples were not cross-contaminated. A sample with $1 \mu 1$ diethylpyrocarbonate-treated water instead of RNA was concomitantly examined for each of the reactions described above. To confirm the amplification specificity, the PCR products were subjected to a melting curve analysis. A positive control using RNA obtained from the HT29 cell line was also performed for each LightCycler run.

Immunoblot. Total protein was extracted from the esophageal carcinoma cell lines using $1 \mathrm{X}$ sample buffer, as described elsewhere (25). Identical aliquots $(30 \mu \mathrm{g})$ of total protein were applied to several $10 \%$ acrylamide gradient gels. After electrophoresis, the samples were electroblotted onto a polyvinylidene difluoride membrane (Immobiline; Millipore, Inc., Bedford, MA) at $0.5 \mathrm{~A}$ for $0.5 \mathrm{~h}$ at $4^{\circ} \mathrm{C}$. Act$\mathrm{BA}$ and MMP-7 were detected using mouse monoclonal primary antibodies to Act- $B A$ (R\&D systems, Minneapolis, $\mathrm{MN}$ ) and MMP7 (Transduction Laboratories, Lexington, KY) at a dilution of 1:1000. The blots were developed using horseradish peroxidase-linked anti-mouse immunoglobulin whole antibody (Promega, Inc., Madison, WI). Signals for Act- $\$ A$ and MMP-7 were detected using Supersignal (Pierce, Inc., Rockford, IL). Pre-stained high molecular weight markers were also run on the gels (Amersham Pharmacia Biotech UK).

cDNA microarray. A cDNA microarray 'Human cancer chip version 2.1' (Takara Biochemicals, Tokyo, Japan) was used and the analysis was performed as follows. For competitive hybridization, cDNAs carrying a Cy5- or Cy3dUTP (Amersham Pharmacia, Piscataway, NJ) label were 
constructed from $15 \mu \mathrm{g}$ of total cellular RNA of control (mock-transfected) cells (KYSE170M) and Act-BA transfectants (KYSE170A1) using an RNA labeling kit (Takara Biochemicals). The microarray was then hybridized to the probes. After overnight hybridization at $65^{\circ} \mathrm{C}$, the slide was twice washed in $2 \mathrm{X} \mathrm{SSC}$ and $0.5 \% \mathrm{SDS}$ for $30 \mathrm{~min}$ at $55^{\circ} \mathrm{C}$, then washed in $2 \mathrm{X} \mathrm{SSC}$ and $0.5 \%$ SDS for $5 \mathrm{~min}$ at $65^{\circ} \mathrm{C}$, and then in $0.05 \mathrm{X} \mathrm{SSC}$ and $0.5 \%$ SDS for $5 \mathrm{~min}$ at room temperature. The slides were then scanned using a GMS418 (Molecular Dynamics, Sunnyvale, CA) with separate measurements of the sample intensities for $\mathrm{Cy} 3$ and $\mathrm{Cy} 5$. The intensity of each hybridization signal was evaluated photometrically using Imagene software (BioDiscovery, Marina Del Rey, CA), and normalized to the average signal of a housekeeping gene, glyceraldehyde-3-phosphate dehydrogenase (G3PDH). The Cy3:Cy5 ratio for each sample was calculated by averaging the spots, and the cut-off value for each expression level was calculated according to the background noise. For this cut-off, we used an expression level of $>100$ where the fluctuation was below a critical value (1.0), because other genes (those with low expression) are embedded in the background noise. Up-regulated and down-regulated genes in the Act- $\beta$ A transfectants were defined as those having a Cy3:Cy5 signal ratio $>2.0$, or $<0.50$, respectively.

Promoter assay. MMP-7 promoters with or without a mutation in the Smad binding site or the AP-1 binding site (established by Crawford et al) were kindly provided by Dr Lynn Matrisian (26). A transfection mixture was created by incubating $1 \mathrm{mg}$ of firefly luciferase reporter with $10 \mathrm{ng}$ of the Renilla luciferase internal control, SV40-RL (Promega), and $1 \mathrm{mg}$ each of the expression vectors indicated below. The volume was brought to $200 \mu 1$ using OptiMEM (Invitrogen), and $15 \mu 1$ of Superfect (Qiagen) transfection reagent was added and mixed by pipetting. After a $15-\mathrm{min}$ incubation, $1 \mathrm{ml}$ of DMEM containing 10\% FBS was added, the contents were mixed, and $400 \mu 1$ was distributed to each of 3 wells of a 24-well plate, each well containing KYSE170M or KYSEA1, 6,9 plated $24 \mathrm{~h}$ prior to transfection. Total DNA in the transfection mixture was kept constant by including the same empty vectors as those that contained the cDNAs being expressed. The transfection mixture was removed from the cells 2-3 h after addition and replaced with DMEM with $10 \%$ FBS. Luciferase activity was determined using the Dual Luciferase kit (Promega) 16-24 h post transfection by lysing in $50 \mu 1$ of passive lysis buffer and assaying both firefly and Renilla luciferase activities in the same $30 \mathrm{ml}$ aliquot of lysate.

Fold-induction was determined by first normalizing each firefly luciferase value to the Renilla luciferase internal control, averaging the normalized values, and dividing by the mean value of the firefly reporter cotransfected with empty vectors only. Normalized relative light units (RLUs) were determined by normalizing each firefly luciferase value to the highest Renilla luciferase value in a given experiment by the following formula: (highest Renilla luciferase value in the experiment/Renilla luciferase value of the individual sample) $x$ firefly luciferase value of the same sample. Whether using fold-induction or normalized RLUs, each experiment was repeated as noted in the figure legends and the means and standard errors were calculated using Microsoft Excel.

Quantitation and statistical methods. The significance of difference between the variables was tested by Student's t-test or Fisher's exact probability test. Correlation between the variables was tested using StatView J-4.5 software (SAS Inst., Cary, NC).

\section{Results}

Activin A expression in Act- $\beta$ A. The open reading frame of Act- $\beta A$ cDNA subcloned in pcDNA 3.1 was transfected into the KYSE170 esophageal carcinoma cell line. We isolated three single clones of Act- $\beta$ A transfected cells (KYSE170A1, $6,9)$ and one single clone of control vector transfected cells (KYSE170M). Expression of Act-BA mRNA was shown by Northern blot (Fig. 1a) and expression of activin A itself was shown by Western blot (Fig. 1b). These results confirmed that the transfection of Act-BA was successful and that the full-length form of activin $A$ was produced by the Act- $\beta A$ transfectants.

Identification of elevated MMP-7 expression in Act $-\beta A$ transfectants. A cDNA microarray analysis was performed on the transfected population-(KYSE170A1) and on control cells (KYSE170M). Tables I and II show the genes which were expressed at higher or lower levels in transfected cells (KYSE 170A1) relative to control cells (KYSE 170M). MMP-7 mRNA expression in KYSE 170A1 showed a 20.17-fold-increase relative to that observed in KYSE170M. The expression of MMP-7 mRNA and protein in three single clones of transfectants (KYSE170A1, A6, A9) was higher than in the control cells (KYSE170M) (Fig. 1a and b).

Correlated expression of Act- $\beta A$ and MMP-7: activin A neutralizing antibody. From the Northern blot and immunoblot, it was apparent that expression of MMP-7 mRNA and protein were higher in transfectants than in control cell lines (Fig. 1a and b). A quantitative reverse transcription polymerase chain reaction (RT-PCR) was performed on mRNA derived from three cloned transfectants and control cells using real-time PCR (Fig. 2a and b). The expression levels of Act- $\beta A$, MMP-7 and G3PDH were calculated relative to their expression in a positive control cell line, HT29. To adjust the expression levels of Act-BA and MMP-7 in each sample, we used the expression levels of G3PDH in the sample and the control, using the following equations: the value for Act- $\beta A$ expression $=\log ($ Act $-\beta A / G 3 P D H)$ while the value for MMP-7 expression = log (MMP-7/ G3PDH), as described previously (25). We then examined the correlation between the values for Act- $B A$ and MMP-7 (Fig. 2c). This result indicates that the expression levels of Act-BA mRNA and MMP-7 mRNA were significantly correlated.

The expression of MMP-7 mRNA in KYSE170A1 was assessed in the presence and absence of activin A neutralizing antibody. The expression of MMP-7 mRNA decreased in the presence of activin A neutralizing antibody in a dose dependent manner, as revealed by Northern blot analysis 
Table I. Higher expression genes in activin A transfectant cells.

\begin{tabular}{|c|c|c|c|c|}
\hline Symbol & Gene name & Protein encorded & Accession no. & Fold \\
\hline MMP7 & Matrix metalloproteinase 7 (matrilysin, uterine) & AAV40839 & AY795972 & 20.2 \\
\hline KRT13 & Keratin 13 & CAA32786 & X14640 & 4.82 \\
\hline CCNG2 & Cyclin G2 & NP_004345 & NM_004354 & 4.69 \\
\hline LTA & Lymphotoxin $\alpha$ (TNF superfamily, member 1$)$ & АAH34729 & ВC034729 & 4.49 \\
\hline TNFSF10 & Tumor necrosis factor (ligand) superfamily, member 10 & NP_003801 & NM_003810 & 4.02 \\
\hline MMP13 & Matrix metalloproteinase 13 (collagenase 3 ) & NP_002418 & NM_002427 & 3.92 \\
\hline KRT6B & Keratin 6B & AAH34535 & $\mathrm{BC} 034535$ & 3.44 \\
\hline TGFBI & Transforming growth factor, $\beta$-induced, $68 \mathrm{kD}$ & AAH04972 & BC004972 & 3.06 \\
\hline CDH8 & Cadherin 8 & NP_001787 & NM_001796 & 2.77 \\
\hline TNFRSF10 & Tumor necrosis factor receptor superfamily, member $10 \mathrm{~b}$ & NP_003833 & NM_003842 & 2.74 \\
\hline COL17A1 & Collagen, type XVI, $\alpha 1$ & NP_000485 & NM_000494 & 2.65 \\
\hline PGK1 & Phosphoglycerate kinase 1 & AAI04838 & BC104837 & 2.54 \\
\hline ALDOA & Aldolase A, fructose-bisphosphate & AAH04333 & BC004333 & 2.51 \\
\hline IFITM2 & Interferon induced transmembrane protein $2(1-8 \mathrm{D})$ & NP_006426 & NM_006435 & 2.43 \\
\hline FDFT1 & Farnesyl-diphosphate farnesyltransferase 1 & AAH29641 & ВC029641 & 2.27 \\
\hline PTGES & Prostaglandin E synthase & AAH08280 & ВC008280 & 2.10 \\
\hline MIHC & Apoptosis inhibitor 1 & AAC83232 & AF070674 & 2.10 \\
\hline SOD2 & Superoxide dismutase 2 , mitochondrial & AAH12423 & BC012423 & 2.08 \\
\hline TGFB1 & Transforming growth factor, $\beta 1$ & AAH22242 & BC022242 & 2.02 \\
\hline TUBB & Tubulin, $\beta$ polypeptide & NP_821133 & NM_178014 & 2.02 \\
\hline TOP2A & Topoisomerase (DNA) II $\alpha(170$ kD) & NP_001058 & NM_001067 & 2.00 \\
\hline
\end{tabular}

Table II. Lower expression genes in activin A transfectant cells.

\begin{tabular}{|c|c|c|c|c|}
\hline Symbol & Gene name & Protein encorded & Accession no. & Fold \\
\hline CASP8 & Caspase 8 , apoptosis-related cysteine protease & NP_001219 & NM_001228 & 0.25 \\
\hline CD8B1 & CD8 antigen, $\beta$ polypeptide 1 (p37) & AAX42824 & AY890886 & 0.31 \\
\hline AREG & Amphiregulin (schwannoma-derived growth factor) & AAX42908 & AY890973 & 0.32 \\
\hline RRAD & Ras-related associated with diabetes & AAH57815 & BC057815 & 0.36 \\
\hline NF2 & Neurofibromin 2 (bilateral acoustic neuroma) & AAH03112 & BC003112 & 0.36 \\
\hline VDR & Vitamin D (1,25-dihydroxyvitamin D3) receptor & NP_000367 & NM_000376 & 0.36 \\
\hline KISS1 & KiSS-1 metastasis-suppressor & NP_002247 & NM_002256 & 0.40 \\
\hline VCAN & Chondroitin sulfate proteoglycan 2 (versican) & NP_004376 & NM_004385 & 0.40 \\
\hline PRDX3 & Peroxiredoxin 3 & AAH59169 & ВC059169 & 0.41 \\
\hline ITGA8 & Integrin, $\alpha 8$ & NP_003629 & NM_003638 & 0.41 \\
\hline CDC16 & CDC16 (cell division cycle 16, S. cerevisiae, homolog) & CAM22287 & AL160396 & 0.44 \\
\hline PIGF & Phosphatidylinositol glycan, class F & NP_775097 & NM_173074 & 0.44 \\
\hline SMN1 & Survival of motor neuron 1 , telomeric & AAH62723 & ВC062723 & 0.48 \\
\hline MKK4 & Mitogen-activated protein kinase kinase 1 & AAC24130 & AH006187 & 0.49 \\
\hline CDC2L5 & $\begin{array}{l}\text { Cell division cycle } 2 \text {-like } 5 \\
\text { (cholinesterase-related cell division controller) }\end{array}$ & CAC10401 & AJ297710 & 0.49 \\
\hline RBL2 & Retinoblastoma-like 2 (p130) & NP_005602 & NM_005611 & 0.49 \\
\hline
\end{tabular}

(Fig. 3a) and real-time PCR (Fig. 3b). These results indicate that the expression of MMP-7 mRNA was attenuated by neutralizing of activin A activity.
The decrease promoter activity of MMP-7 promoter with mutation of ap-1 binding site. We assessed the promoter activity of the human MMP-7 promoter (HMAT) in 
a

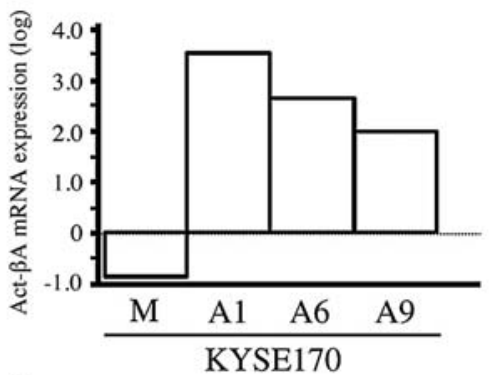

b
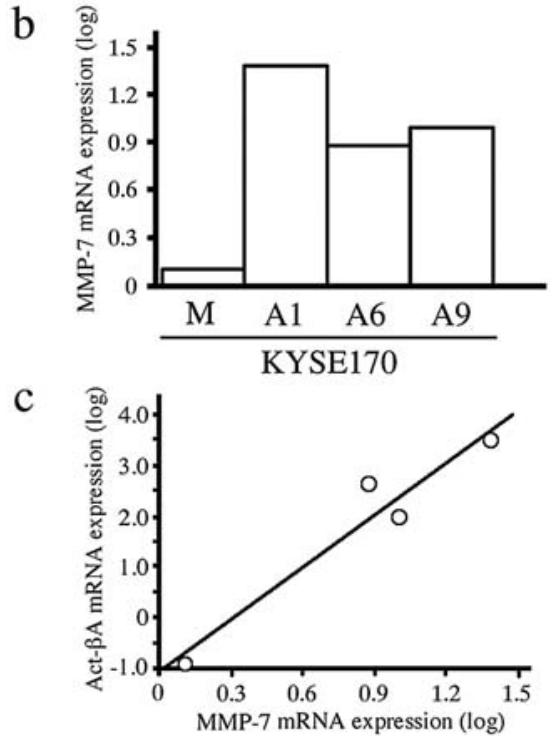

Figure 2. Expression of Act-BA mRNA and MMP-7 mRNA. Expression was assessed by the quantitative reverse transcription polymerase chain reaction method. (a) The relative expression of Act-BA mRNA in KYSE170M1, A1, 6 and 9 cells compared to control cells (KYSE 70). (b) The relative expression of MMP-7 mRNA in KYSE170M1, A1, 6 and 9 cells compared to control cells (KYSE70). (c) The correlation between Act-BA and MMP-7 expression. The correlation coefficient is 0.693 .

KYSE170A1, A6, A9 and KYSE170M. Promoter activity was elevated in Act-BA transfectants compared with control cells (Fig. 4a). To identify the transcription factor that was modulated by activin A, we used HMAT promoters with a mutation in either the Smad binding site or the AP-1 binding site. The promoter activities of HMATs with wild-type or mutant of Smad binding sites were similar in Act-BA transfectants and control cells (KYSE170A1). However, the promoter activity of HMAT with a mutant of AP-1 binding site was lower than that of wild-type HMAT (Fig. 4b).

Nuclear accumulation of $c$-Jun in Act- $\beta$ A transfectants. To evaluate the expression of c-Jun and Smad2 in Act-BA transfectants (KYSE170A1) and control cells (KYSE170M), we performed immunofluorescent staining and immunoblot analysis. The immunofluorescent stain revealed nuclear accumulation of Smad2/3 and higher expression of c-Jun in nuclei inKYSE170A1 (Fig. 5a). In the immunoblot analysis, the amount of Smad2/3 in nuclei of Act- $\beta A$ transfectants (KYSE170A1, 6, 9) was elevated, but the total amount of Smad2/3 was similar to that of control cells (KYSE170M) (Fig. 5b). Furthermore, c-Jun in Act-BA transfectants (KYSE170A1, 6, 9) was expressed at higher levels than in control cells (KYSE170M) (Fig. 5b). These results indicate a

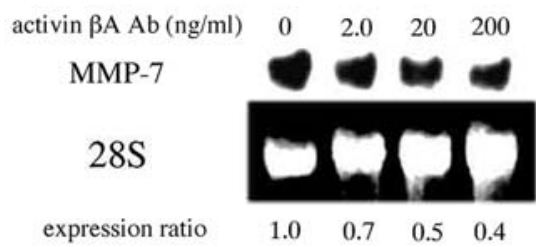

b

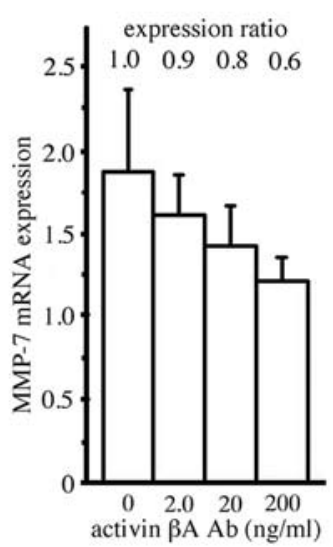

Figure 3. Effect of anti-human activin A neutralizing antibody on KYSE170A1. Expression of MMP-7 mRNA was inhibited in a dosedependent fashion by activin A neutralizing antibody. (a) Northern blotting, upper number indicates the dose of anti-human activin A neutralizing antibody and lower number indicates the mRNA expression ratio compared to KYSE170M. (b) Quantitative RT-PCR, upper number indicates the expression ratio compared to KYSE170M.
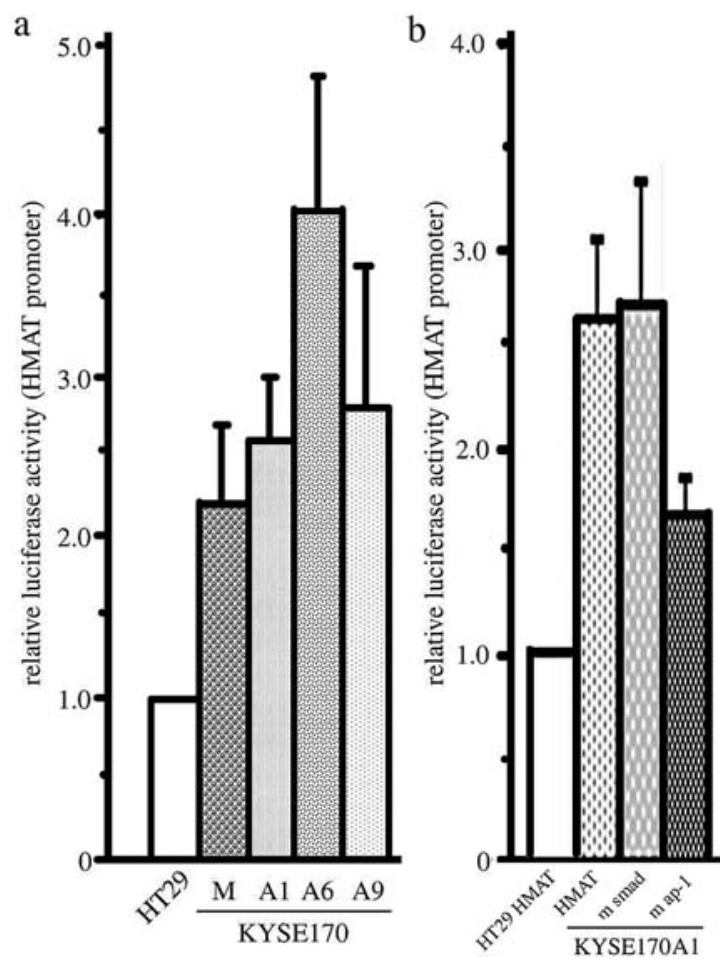

Figure 4. The reporter assay for human MMP-7. (a) The relative luciferase activities in KYSE170M, A1, 6, 9 and HT29 are assessed. Cloned Act-BA transfectants (KYSE170A1, 6 and 9) possessed higher luciferase activities than KYSE170M. (b) Evaluation of the promoter activity of MMP-7 with or without mutation of the Smad binding site or the AP-1 binding site. The luciferase activity of the MMP-7 promoter with a mutant of AP-1 binding site is reduced compared to the promoter activity of MMP-7 with or without mutation in Smad binding site. 
a
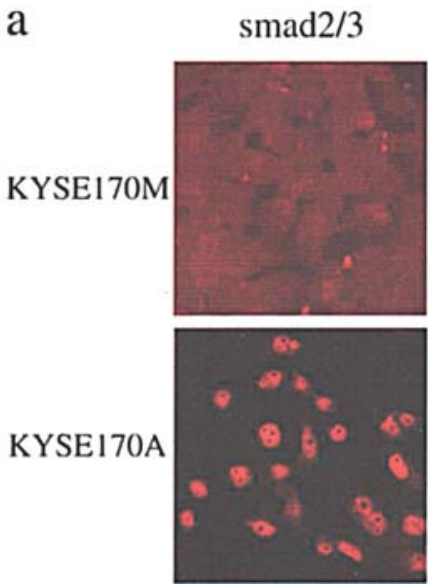

c-Jun
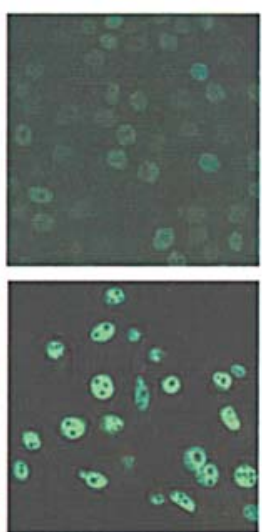

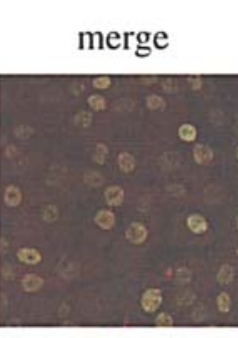

b

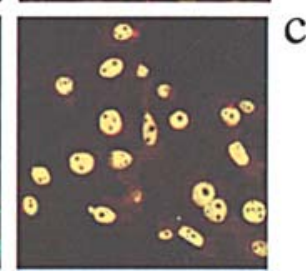

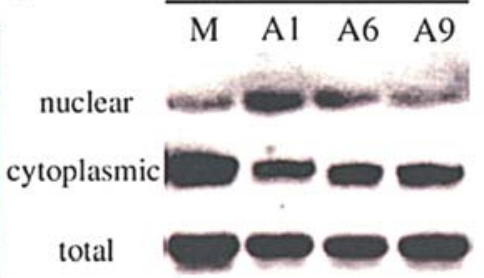

C

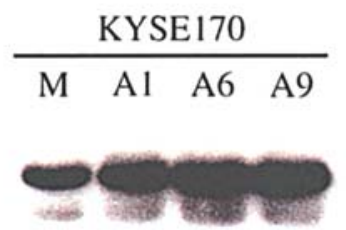

Figure 5. Effect of activin A on specific transcription factors. (a) Immunofluorescent staining of Smad2/3 and c-Jun in KYSE170M and A1. Strong nuclear stain of Smad2/3 and c-Jun was observed in KYSE170A1 compared to KYSE170M. (b) Immunoblot of Smad2/3 was performed using total, cytoplasmic or nuclear protein extracts of KYSE170M, A1, 6 and 9. In all Act-ßA transfectant clones (KYSE170A1, 6, 9), nuclear Smad2/3 is abundant compared to KYSE170M. (c) Immunoblot analysis of c-Jun. The expression of c-Jun is higher in KYSE170A1, 6 and 9 than in KYSE170M.

that activin A mediates the expression of c-Jun in nuclei and may enhance the promoter activities of wild-type HMAT.

\section{Discussion}

The elevated expression of activin A in solid carcinomas is related to cancer aggressiveness. To reveal this mechanism, we performed cDNA microarray analysis using mRNA samples from stable activin- $\beta A$ (Act- $\beta A$ ) transfectant cells (KYSE170A1) and mock control cells (KYSE170M). The cDNA microarray analysis showed that MMP-7 expression was elevated in KYSE170A1. We showed that the MMP-7 expression level was directly correlated with the level of the Act- $\beta A$ expression in transfectants and control cells. Furthermore, MMP-7 expression in KYSE170A1 was attenuated by anti-human activin A neutralizing antibody. These results indicated that activin A augments MMP-7 expression.

We assumed that the promoter activity of HMAT was not strong. However, it was possible to evaluate the luciferase activities in HT29, KYSE170M, A1, A6 and A9. The luciferase activities of all transfectant clones were greater than that of KYSE170M. However, values were somewhat low and statistical significance could not be demonstrated. The reporter assay showed that there was no remarkable difference between the promoter activity of MMP-7 with or without a mutation of the Smad binding site. However, the difference was observed in MMP-7 promoter activity when the AP-1 binding site was altered. Several reports have shown that activin A and/or TGF- $\beta$ modulate c-Jun expression $(27,28)$ and that of c-Jun/AP-1 regulates MMP-7 expression (29-31). In the immunofluorescence assay, the c-Jun signal was higher in the transfectants compared to control cells, but a faint c-Jun signal was observed in the control cells. These results indicate that while activin A may not be absolutely required for MMP-7 expression, it does appear to enhance MMP-7 expression through the AP-1 binding site. MMP-7 expression in solid carcinomas is associated with the cancer aggressiveness. Our data may indicate that MMP-7 expression mediated by activin A plays one of the important roles of esophageal carcinoma aggressiveness.

\section{Aknowledgements}

This work was supported in part by the following grants and foundations: a Grant-in-Aid for Scientific Research on Priority Areas of Cancer (12215116, 11671251, 12218227), a Grant-in-Aid for Scientific Research (B) (12557100, 12470241) and (C) $(12213101,12671232,12670166)$, and a Grant-in-Aid for Exploratory Research (13877188), from the Ministry of Education, Culture, Sports, Science and Technology of Japan; and by the Uehara Memorial Foundation, the Naito Foundation, the Casio Science Promotion Foundation, the Foundation for Promotion of Cancer Research in Japan, and the Sagawa Foundation for Promotion of Cancer Research. We are grateful to Ms. K. Ogata and Ms. T. Shimooka for excellent technical assistance.

\section{References}

1. Yoshinaga K, Mimori K, Yamashita K, Utsunomiya T, Inoue H and Mori M: Clinical significance of the expression of activin A in esophageal carcinoma. Int J Oncol 22: 75-80, 2003.

2. Kleeff J, Ishiwata T, Friess H, Buchler MW and Korc M: Concomitant over-expression of activin/inhibin beta subunits and their receptors in human pancreatic cancer. Int J Cancer 77: 860-868, 1998.

3. Thomas TZ, Wang H, Niclasen P, et al: Expression and localization of activin subunits and follistatins in tissues from men with high grade prostate cancer. J Clin Endocrinol Metab 82: 3851-3858, 1997.

4. Zheng W, Luo MP, Welt C, et al: Imbalanced expression of inhibin and activin subunits in primary epithelial ovarian cancer. Gynecol Oncol 69: 23-31, 1998.

5. Wildi S, Kleeff J, Maruyama H, Maurer CA, Buchler MW and Korc M: Overexpression of activin A in stage IV colorectal cancer. Gut 49: 409-417, 2001.

6. Petraglia F, Florio P, Luisi S, et al: Expression and secretion of inhibin and activin in normal and neoplastic uterine tissues. High levels of serum activin A in women with endometrial and cervical carcinoma. J Clin Endocrinol Metab 83: 1194-1200, 1998.

7. Kojima I and Ogata E: Dual effect of activin A on cell growth in Balb/c 3T3 cells. Biochem Biophys Res Commun 159: 1107-1113, 1989. 
8. Rabinovici J, Spencer S and Jaffe S: Recombinant human activin-A promotes proliferation of human luteinized preovulatory granulosa cells in vitro. J Clin Endocrinol Metab 71: 1396-1398, 1990.

9. Di Simone N, Crowley WF Jr, Wang QF, Sluss PM and Schneyer AL: Characterization of inhibin/activin subunit, follistatin and activin type II receptors in human ovarian cancer cell lines: a potential role in autocrine growth regulation. Endocrinology 137: 486-494, 1996.

10. Coerver KA, Woodruff TK, Finegold MJ, Mather J, Bradley A and Matzuk MM: Activin signaling through activin receptor type II causes the cachexia-like symptoms in inhibin-deficient mice. Mol Endocrinol 10: 534-543, 1996.

11. Matrisian LM: The matrix-degrading metalloproteinases. BioEssays 14: 455-463, 1992.

12. Imai K, Yokohama Y, Nakanishi I, et al: Matrix metalloproteinase 7 (matrilysin) from human rectal carcinoma cells. Activation of the precursor, interaction with other matrix metalloproteinases and enzymatic properties. J Biol Chem 270; 6691-6697, 1995.

13. Murphy G, Cocket M, Ward R and Docherty AJP: Matrix metalloproteinase degradation of elastin, type IV collagen and proteoglycan: a quantitative comparison of the activities of 95 and $72 \mathrm{kDa}$ gelatinases, stromelysin- 1 and -2 and punctuated metalloproteinase (PUMP). Biochem J 277: 277-279, 1991.

14. Sires UI, Griffin GL, Broekelmann TJ, et al: Degradation of entactin by matrix metalloproteinases. Susceptibility to matrilysin and identification of cleavage sites. J Biol Chem 268: 2069-2074, 1993.

15. Crabbe T, Smith B, O'Connell J and Docherty A: Human progelatinase $A$ can be activated by matrilysin. FEBS Lett 345: 14-16, 1994.

16. Sang QA, Bodden MK and Windsor LJ: Activation of human progelatinase A by collagenase and matrilysin: activation of procollagenase by matrilysin. J Protein Chem 15: 243-253, 1996.

17. Sires UI, Murphy G, Baragi VM, Fliszar CJ, Welgus HG and Senior RM: Matrilysin is much more efficient than other matrix metalloproteinases in the proteolytic inactivation of a1antitrypsin. Biochem Biophys Res Commun 204: 613-620, 1994.

18. Mori M, Barnard GF, Mimori K, Ueo H, Akiyoshi T and Sugimachi K: Overexpression of matrix metalloproteinase-7 mRNA in human colon carcinomas. Cancer 75: 1516-1519, 1995.

19. Honda M, Mori M, Ueo H, Sugimachi K and Akiyoshi T: Matrix metalloproteinase-7 expression in gastric carcinoma. Gut 39: 444-448, 1996.
20. Yamashita K, Mori M, Shiraishi T, Shibata K and Sugimachi K: Clinical significance of matrix metalloproteinase-7 expression in esophageal carcinoma. Clin Cancer Res 6: 1169-1174, 2000.

21. Gaire M, Magbanua Z, McDonnell S, McNeil L, Lovett DH and Matrisian LM: Structure and expression of the human gene for the matrix metalloproteinase matrilysin. J Biol Chem 269: 2032-2040, 1994.

22. Mori M, Shiraishi T, Tanaka S, et al: Lack of DMBT1 expression in oesophageal, gastric and colon cancers. Br J Cancer 79: 211-213, 1999.

23. Mori M, Mimori K, Inoue H, et al: Detection of cancer micrometastases in lymph nodes by reverse transcriptase-polymerase chain reaction. Cancer Res 55: 3417-3420, 1995.

24. Sugimachi K, Matsuura H, Kai H, et al: Prognostic factors of esophageal carcinoma: univariate and multivariate analyses. J Surg Oncol 31: 108-112, 1986.

25. Yoshinaga $\mathrm{K}$, Inoue $\mathrm{H}$, Utsunomiya $\mathrm{T}$, et al: $\mathrm{N}$-cadherin is regulated by activin $\mathrm{A}$ and associated with tumor aggressiveness in esophageal carcinoma. Clin Cancer Res 10: 5702-5707, 2004.

26. Crawford H, Fingleton B, Gustavson M, et al: The PEA3 subfamily of Ets transcription factors synergizes with beta-cateninLEF-1 to activate matrilysin transcription in intestinal tumors. Mol Cell Biol 21: 1370-1383, 2001.

27. Tardif G, Reboul P, Dupuis M, et al: Transforming growth factor-beta induced collagenase-3 production in human osteoarthritic chondrocytes is triggered by Smad proteins: cooperation between activator protein-1 and PEA-3 binding sites. J Rheumatol 28: 1631-1639, 2001.

28. Norwitz ER, Xu S, Xu J, et al: Direct binding of AP-1 (Fos/Jun) proteins to a SMAD binding element facilitates both gonadotropin-releasing hormone (GnRH)- and activin-mediated transcriptional activation of the mouse GnRH receptor gene. J Biol Chem 277: 37469-37478, 2002.

29. Le Floch N, Rivat C, De Wever O, et al: The proinvasive activity of Wnt-2 is mediated through a non-canonical Wnt pathway coupled to GSK-3beta and c-Jun/AP-1 signaling. FASEB J 19: 144-146, 2005.

30. Rivat C, Le Floch N, Sabbah M, et al: Synergistic cooperation between the AP-1 and LEF-1 transcription factors in activation of the matrilysin promoter by the src oncogene: implications in cellular invasion. FASEB J: 1721-1723, 2003.

31. Pacheco MM, Kowalski LP, Nishimoto IN and Brentani MM: Differential expression of c-Jun and c-Fos mRNAs in squamous cell carcinoma of the head and neck: associations with uPA, gelatinase B and matrilysin mRNAs. Head Neck 24: 24-32, 2002. 